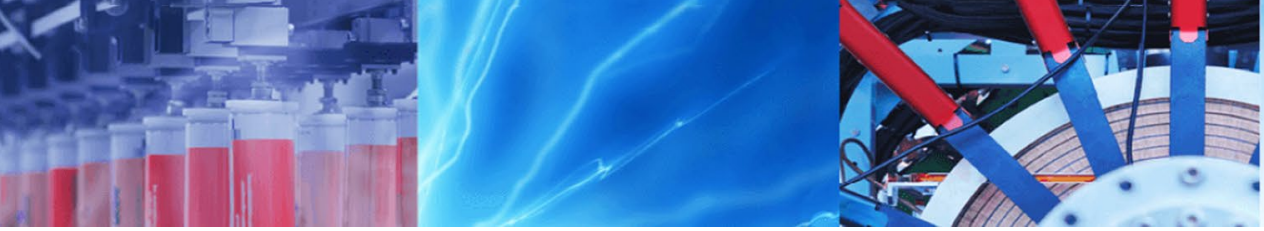

Research Article

\title{
Effect of ZTA concentration on structural, thermal, mechanical and dielectric behavior of novel ZTA-PVA nanocomposite films
}

\author{
Chaitra Srikanth ${ }^{1}$. G. M. Madhu ${ }^{1}$ (1)
}

Received: 17 December 2019 / Accepted: 10 February 2020 / Published online: 17 February 2020

(c) Springer Nature Switzerland AG 2020

\begin{abstract}
Zirconia toughened alumina (ZTA) nanopowder was synthesized by solution combustion technique. Polymer nanocomposites were prepared using ZTA nanopowder as the reinforcement and polyvinyl alcohol (PVA) as the matrix. ZTA nanopowder composition was varied from 0 to $2.5 \%$ by weight. Structural characterization was done using scanning electron microscope (SEM) and X-ray diffraction (XRD). XRD results showed prominent, well defined peaks of zirconia and a-alumina, hence confirmed that ZTA is a crystalline material. SEM images showed that, level of agglomeration kept increasing due to increase in filler content, which might have contributed to film stiffness. Thermal analysis was carried out using differential scanning calorimetry. Addition of ZTA into PVA matrix resulted in increase in melting point as well as glass transition temperature. Influence of the nanofiller concentration on the electrical conductivity was found using Agilent 4249A impedance analyser. Conductivity measurements were carried out for all the nanocomposite films doped with ZTA and were found to exhibit insulating properties. Change in mechanical properties such as Young's modulus, tensile strength and film toughness of PVA films as a function of nano filler content are reported.
\end{abstract}

Keywords Polymer nanocomposites - ZTA · Conductivity $\cdot$ Young's modulus · Tensile strength

\section{Introduction}

Alumina is a popular ceramic known for its structural and technological applications. Structural properties of alumina are governed by its matrix stability and microstructure $[1,2]$. Ceramic materials are generally compounds that exhibit electrical insulating properties and chemically inert towards acids, alkalis, organic and inorganic compounds $[3,4]$. Alumina in particular shows good mechanical and wear resistance, gas tight, high compressive strength under extreme temperatures, corrosive atmospheres and excellent biocompatibility [5, 6]. Zirconia is a trending ceramic material in the manufacture of thin films. It has gained attention due to its different attractive properties such as chemical inertness to oxidative atmospheres, very low electrical conductivity, thermal shock resistance and biocompatibility. Excellent mechanical properties were observed such as Young's modulus similar to that of stainless steel, hardness, wear resistance, mechanical strength and frictional resistance even at elevated temperatures. Thus, zirconia is a suitable candidate for various applications such as thermal barrier coatings, refractories, pigments, buffer layers for superconductors, piezoelectric devices, ceramic condensers, fuel cells, electrode and oxygen sensors, orthopaedic implants and most importantly catalysts [7-9].

Alumina ceramic is a brittle material exhibiting moderate fracture toughness and limited flexural strength as compared to zirconia. In this regard, alumina ceramics may pose a potential catastrophic failure of products in stress induced environments [10,11]. Incorporation of tetragonal zirconia as a second phase into alumina is of

G. M. Madhu, gmmadhu@gmail.com|'Department of Chemical Engineering, M S Ramaiah Institute of Technology, MSRIT Post, MSR Nagar, Bangalore, Karnataka 560054, India. 
our particular interest and it is called as Zirconia Toughened Alumina (ZTA). ZTA nullifies many disadvantages incurred by alumina and incorporates the benefits of both alumina and zirconia ceramics. Hence, it can be categorised as advanced ceramic material with special properties such as, high hardness and low brittleness, high fracture toughness, high strength and high ageing resistance. These properties make ZTA material of choice to work under high stress induced environments such as extreme mechanical abrasion and thermal shock $[10,12]$.

Many researchers have reported different methods of fabrication of nano ZTA. Tulliani et al. [13] synthesized alumina-zirconia ceramic foams by gel-casting-fugitive phase process using agar as gelling agent and polyethylene as pore formers. These polymer composites exhibited porosity as high as $75 \mathrm{vol} \%$. Mechanical characterization of these samples showed that, compressive strength decreased with decrease in relative density and increased with increase in porosity. Nagashima et al. [14] fabricated $\mathrm{Al}_{2} \mathrm{O}_{3}-5 \% \mathrm{ZrO}_{2}$ by high energy ball milling process using $\mathrm{Al}_{2} \mathrm{O}_{3}$ powder and $\mathrm{Zr}$ alkoxide as precursors. Micro and nano composite powders were synthesized by this technique and the effect of different sintering temperatures on the microstructure were investigated. They found that, during the production of intra-granular particles, $\mathrm{ZrO}_{2}$ particles played a major role in growing alumina grains during the sintering process. Xin et al. [15] synthesized micro-nano ZTA composite by multiphase flocculating suspension method. The micro-nano ZTA composed of micro-alumina and nano zirconia particles. Two interesting phenomena of the composite were observed. Firstly, low zirconia content in the composite (10 vol\%) showed trans-granular fracture due to fine zirconia nanoparticles embedded inside alumina grains. High zirconia content (20 vol\%) in the composite exhibited better toughness and better resistance towards crack propagation. Sarkar et al. [16] fabricated nano ZTA by sol gel method using $\mathrm{Al}\left(\mathrm{NO}_{3}\right)_{3} \cdot 9 \mathrm{H}_{2} \mathrm{O}$ and $\mathrm{ZrOCl}{ }_{2} \cdot 8 \mathrm{H}_{2} \mathrm{O}$ as precursors. The results revealed that, incorporation of $\mathrm{t}-\mathrm{ZrO} 2$ in alumina matrix resulted increase in hardness and toughness values. This motivated us to use ZTA as a nano filler in our research work.

Polyvinyl alcohol (PVA) is a most preferred material of choice as a host matrix by various researchers [17-22] for the synthesis of polymer nanocomposites due its water soluble, non-toxic, ease of fabrication, transparent film forming ability, biodegradability, chemical stability and interesting mechanical properties. PVA is a semi-crystalline polymer with high optical clarity [20]. The hydroxyl bond present in PVA is found to exhibit good interaction between the matrix and the nanoparticles due its high aspect ratio [20]. It is found to exhibit high dielectric permittivity with very good insulating properties hence, it is extensively used in electronic devices [22]. It is also used in textile and food industries. Hence it is used as a host matrix in our research work.

Some researchers have reported work on incorporating alumina into PVA matrix. Suzuki et al. [23] have synthesized alumina particles $(<500 \mathrm{~nm})$ by sol gel method and investigated its influence on PVA films. They found that there was a slight increase in the tensile strength, and a profound increase in Young's modulus values of the PVA-alumina films as compared to plain PVA films. However, the increase in alumina content had a detrimental effect on the film toughness and drastic decrease in the tensile strain values. This might have been due to the size of crystallite dispersed in the PVA matrix [23]. Panda et al. [24] synthesized alumina nano fibres by electrospinning technique using aluminium nitrate as a precursor. Different concentrations of alumina nanopowder was reinforced in PVA solution and its effect on sintering temperatures were studied. The morphological details were studied using SEM and XRD was used to find the diameter of the crystallites.

Most of the research so far has been focusing upon fabrication of ZTA nanoparticles by various methods and used for a wide range of applications. So far no work has been reported on ZTA and PVA based polymer nanocomposite films which makes the current work interesting. The present work aims at synthesis of ZTA by solution combustion method [12], and to study the on effect of filler concentration on PVA films for applications in areas which demand insulation properties with high tensile strength and Young's modulus. The samples were characterized for structural properties using scanning electron microscope (SEM) and X-ray diffractometer (XRD). Energy dispersive $X$-ray (EDX) analysis was used to analyse the chemical composition. Thermal characterization was done using differential scanning calorimetry (DSC). Electrical conductivity measurements for all the nanocomposite films were carried out using Agilent 4249A impedance analyser. All the measurements related to mechanical properties such as Young's modulus, ultimate tensile strength and film toughness were made using Universal Testing Machine (UTM). The performance of PVA-ZTA films for the above mentioned testing parameters are analysed and reported.

\section{Experimental}

\subsection{Reagents and materials}

Zirconyl nitrate (99.5\% purity) was procured from M/S Loba Chemie laboratory reagents and fine chemicals having a molecular weight of $231.23 \mathrm{~g} / \mathrm{mol}$. Aluminium nitrate nonahydrate crystals ( $98.5 \%$ purity), having a molecular 
weight of $375.13 \mathrm{~g} / \mathrm{mol}$ was procured from M/S Merck specialities private limited. Urea having a molecular weight of $60.06 \mathrm{~g} / \mathrm{mol}$ was obtained from S D Fine-Chem limited. PVA powder having a molecular weight of $14,000 \mathrm{~g} / \mathrm{mol}$ was procured from $\mathrm{M} / \mathrm{s}$. Thomas Baker chemicals. Urea was used as a fuel for the solution combustion reaction to take place. Double distilled water was used for the preparation of reaction mixtures and casting of thin films using PVA.

Solution combustion technique was used for the synthesis of ZTA nano powders since, it is a relatively inexpensive and most beneficial method to synthesize nanomaterials in its purest forms at low temperatures. Zirconyl nitrate and aluminium nitrate were used as metal oxidizers and urea was used as a fuel to propagate the reaction. Stoichiometric quantities of the reaction mixture [12] were taken i.e., $16.6 \mathrm{~g}$ of aluminium nitrate, $3.25 \mathrm{~g}$ of zirconyl nitrate and $17.8 \mathrm{~g}$ of urea were dissolved in $30 \mathrm{ml}$ of double distilled water. The reaction mixture was kept in a preheated muffle furnace and heated to $400^{\circ} \mathrm{C}$ for $45 \mathrm{~min}$ [12]. Self-ignition of the reaction mixture resulted in the formation of a soft, porous, pure white ZTA nano powder, which had a foamy appearance. The nanopowder was later crushed softly and sintered in the muffle furnace. The calcination temperature and duration were $600{ }^{\circ} \mathrm{C}$ and $1 \mathrm{~h}$ respectively. The overall reaction can be represented as shown in Eq. (1) [12]:

$$
\begin{aligned}
& 8 \mathrm{Al}\left(\mathrm{NO}_{3}\right)_{3}+\mathrm{Zr}\left(\mathrm{NO}_{3}\right)_{2}+24 \mathrm{CH}_{4} \mathrm{~N}_{2} \mathrm{O}+0.5 \mathrm{O}_{2} \\
& \quad \longrightarrow\left(4 \mathrm{Al}_{2} \mathrm{O}_{3}\right)-\mathrm{ZrO}_{2}+24 \mathrm{CO}_{2}+47 \mathrm{H}_{2} \mathrm{O}+38 \mathrm{~N}_{2}
\end{aligned}
$$

As indicated by the above reaction, large volumes of gases such as nitrogen, carbon dioxide and water vapour were released as the reaction commenced. It can be concluded that, to obtain one mole of ZTA, $8 \mathrm{~mol}$ of aluminium nitrate, one mole of zirconyl nitrate and $24 \mathrm{~mol}$ of urea was required along with $0.5 \mathrm{~mol}$ of excess oxygen.

\subsection{Fabrication of polymer nanocomposites}

Thin films were cast using solution intercalation technique using PVA. The technique involved preparing PVA solution of $7.4 \mathrm{wt} \%$ by dissolving PVA powder $(37.9 \mathrm{~g}$ ) in double distilled water $(500 \mathrm{ml})$ by constantly heating at $65-70^{\circ} \mathrm{C}$ for $6 \mathrm{~h}$ using a temperature controlled heater and a magnetic stirrer. At the end of $6 \mathrm{~h}$, clear PVA solution was obtained, which was cooled to room temperature. The solution was further ultra-sonicated for $45 \mathrm{~min}$ at $200 \mathrm{~W}$ and later transferred on to a glass substrate to cast thin films. The casted thin films were left undisturbed for 3 days in a clean, dust free environment, care was taken to avoid bubble formation in the films. Once the pure PVA thin film was obtained, polymer nanocomposite films were cast with different concentrations i.e., $0.5 \%, 1 \%, 1.5 \%, 2 \%$ and $2.5 \%$ of ZTA in PVA. Further increase in ZTA concentration in PVA led to agglomerated molecules in PVA due to non-uniform distribution of the filler in the matrix. Hence, results up to $2.5 \mathrm{wt} \%$ concentration of ZTA in PVA are reported. Thickness measurements for all the polymer composite films were done using digital Vernier callipers. The film thickness ranged between 0.18 and $0.2 \mathrm{~mm}$.

\subsection{Measurements}

It is essential to carry out preliminary characterization of samples to analyse their morphological details, microstructure, chemical composition, phase transformations and thermal behaviour. Hence, certain characterization tools such as SEM with EDX, XRD and DSC were used to analyse the polymer nanocomposite films. SEM analysis was done using Carl Zeiss Ultra 55 model at an operating voltage of $5 \mathrm{kV}$. EDX was carried out using ZEISS EDS detector. XRD analysis was carried out using Rigaku SmartLab powder/thin film X-ray diffractometer. Cu-ka (40kv, $30 \mathrm{~mA})$ was the filter/X-ray source. Scan speed/duration time was found to be $6.0^{\circ} / \mathrm{min}$ and the scan range was from 5 to $80^{\circ}$. Dielectric measurements of thin films were made using Cascade Microtech PM5 Key Sight B1500A semiconductor analyser along with high frequency impedance analyser (Agilent 4294A) was used for electrical characterization of polymer composite films and the frequency ranged from $1 \mathrm{kHz}$ to $5 \mathrm{GHz}$. Thermal analysis was carried out using Universal DSC Q200 V24.11 Build 124 Instrument with a temperature ranging from 30 to $300^{\circ} \mathrm{C}$. Heating and cooling rates were maintained at $10^{\circ} \mathrm{C} \cdot \mathrm{min}^{-1}$. Mechanical properties were studied using Universal Testing Machine (UTM). All the tensile tests were performed as per ASTM D638 standard procedure. Load cell was set at $0.2 \mathrm{KN}$. Film thickness ranged from 0.18 to $0.2 \mathrm{~mm}$. Film width ranged from 13.5 to $14 \mathrm{~mm}$. The error range of UTM was found to be $\pm 0.2 \mathrm{MPa}$. Film thickness and width measurements were made using digital Vernier Calipers.

\section{Results and discussion}

\subsection{XRD}

XRD analysis showed that zirconia existed in tetragonal phase whereas, a-alumina existed in hexagonal phase. Sharp peak at $30.18^{\circ}$ was observed along with many symmetrical and distinctive peaks, which indicated that the ZTA nanopowder is a crystalline material. $\mathrm{Al}_{2} \mathrm{O}_{3}$ diffraction peaks were observed at $2 \theta$ values of $25.57^{\circ}, 28.24^{\circ}$, $37.65^{\circ}, 43.33^{\circ}, 52.55^{\circ}, 57.47^{\circ}, 66.66^{\circ}$ and $74.53^{\circ}$ which are associated with (l $\left.\begin{array}{lll}0 & 1\end{array}\right),\left(\begin{array}{llll}1 & 0 & 1\end{array}\right),\left(\begin{array}{lll}1 & 1 & 0\end{array}\right),\left(\begin{array}{lll}1 & 1 & 3\end{array}\right),\left(\begin{array}{lll}0 & 2 & 4\end{array}\right),\left(\begin{array}{ll}1 & 1\end{array}\right.$ 6), (2 14 ) and (2 20 ) planes respectively. $\mathrm{ZrO}_{2}$ diffraction 
peaks were observed at $2 \theta$ values of $30.18^{\circ}, 34.48^{\circ}, 35.21^{\circ}$, $50.15^{\circ}, 50.70^{\circ}, 59.15^{\circ}, 60.12^{\circ}$ and $62.72^{\circ}$ which are associ-

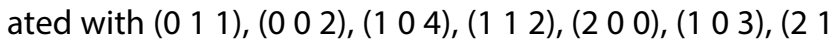
1) and (2 02 ) planes respectively as shown in Fig. 1a. The peak splitting at $50.15^{\circ}$ and $50.70^{\circ}$ confirmed that zirconia is existing in tetragonal phase [25]. Similar peaks positions were observed by different authors who synthesized ZTA by various routes $[25,26]$. The peak positions were in good agreement with JCPDS file number: 98-006-6787 for t-zirconia and JCPDS file number: 98-008-5137 for a-alumina. Debye-Scherrer formula was used to calculate the size of the crystal [16] as shown in Eq. (2) below.

$\mathrm{t}=0.9 \lambda / \beta \cos \theta$

where $\lambda$ is the wavelength of the $x$-ray source used $(0.15418 \mathrm{~nm}$ for Cu k-alpha), $\beta$ is the full width at half maximum and $\theta$ is the angle of diffraction. The smallest crystal size was found to be $9.17 \mathrm{~nm}$ and largest crystal was $112.89 \mathrm{~nm}$. Total average particle size was found to be $39.6772 \mathrm{~nm}$.

Figure $1 \mathrm{~b}$ shows the XRD data of pure PVA and PVA films doped with varying concentrations of ZTA from 0.5 to $2.5 \%$ by weight. One common, yet significant peak was observed among all the samples at $2 \theta=19.4^{\circ}$, this diffraction peak indicated the presence of PVA associated with (1 $\left.\begin{array}{ll}0 & 1\end{array}\right)$ plane $[7,22]$. The crystal lattice orientation of PVA indicates that it is a semi-crystalline polymer. The semicrystalline nature may be attributed due to strong interaction among the polymer groups present in PVA [19]. The peak at $2 \theta=19.4^{\circ}$ remained prominent in all the polymer composite films. When ZTA was doped into PVA, additional sharp peaks kept on increasing. Similar peak positions of zirconia and alumina were present in the polymer nano composite film. The increase in the filler content contributed to prominent peaks of zirconia and alumina in the polymer nano composite films. This could be due to transition of the polymer from semi-crystalline to crystalline compound with increase in doping concentration of ZTA in PVA matrix.

\subsection{SEM and EDX}

Surface morphological features of the synthesized ZTA nanopowder, pure PVA films and PVA films doped with varying concentrations of ZTA were found using SEM. SEM image of ZTA nanopowder is as shown in Fig. 2. Each particle appears to be spherical in shape and uniformly distributed. The smallest particle appears to be $38.47 \mathrm{~nm}$ and the agglomerated particles appear to be around $577.6 \mathrm{~nm}$ in size. Since there is high surface energy existing between particles, this contributes to particle agglomeration and hence resulting in particles of different sizes within the
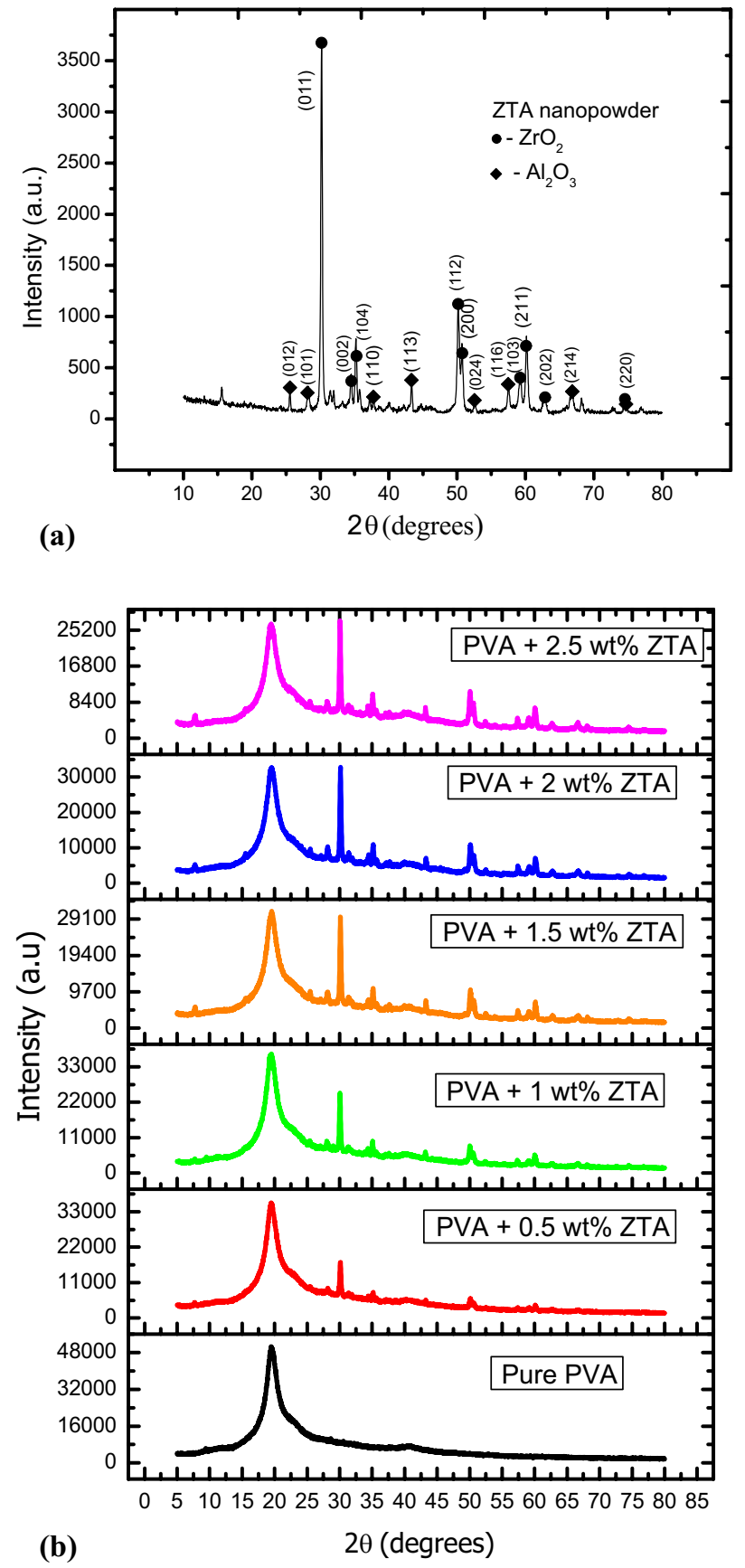

Fig. 1 a XRD pattern of ZTA nano powder. $\mathbf{b}$ XRD pattern of pure PVA film and ZTA nano powder doped PVA thin films

same nanopowder [21]. Figure 3a shows image of pure PVA with uniform distribution of PVA molecules whereas, the varying concentrations of ZTA in PVA is shown in Fig. $3 b, c, d$, e and $f$. The level of agglomeration of ZTA nano particles keep on increasing with increase in concentration in PVA films. Since there is a density difference between PVA and ZTA nano particles, the agglomeration is more prominent at the corner of these films. One such 


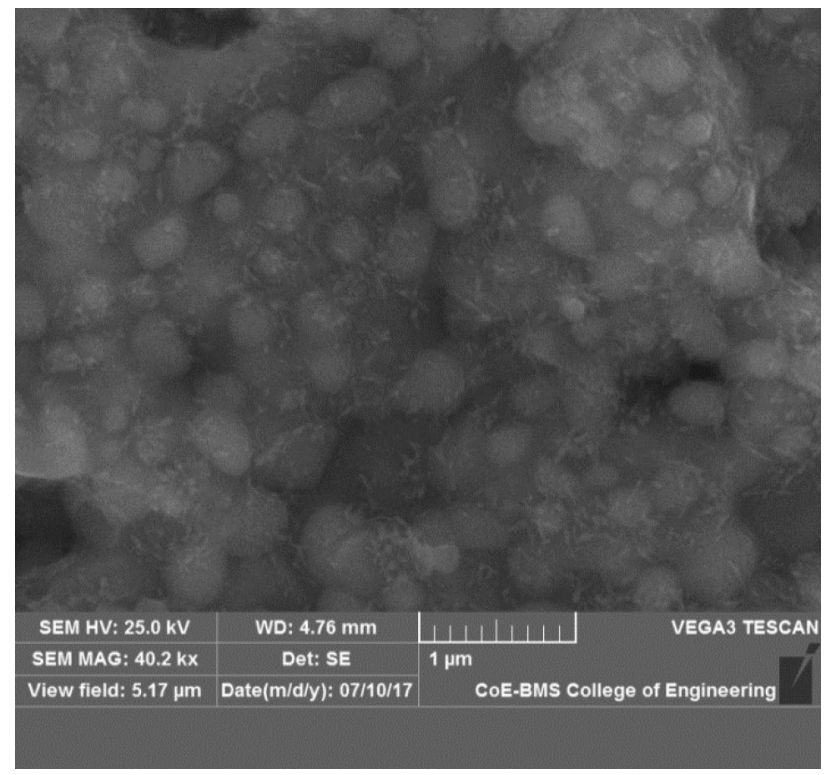

Fig. 2 SEM image of ZTA nano powder (40,200X)

agglomerated cluster is magnified and depicted for PVA with 2.5 wt\% ZTA concentration (Fig. 3f). ZTA particle size ranged from 50 to $600 \mathrm{~nm}$ in the polymer nanocomposite films. This value closely matched the results obtained from XRD data obtained using Debye-Scherrer formula. The agglomerated cluster size ranged from $500 \mathrm{~nm}$ to $13.96 \mu \mathrm{m}$ in the polymer nanocomposite films. As the ZTA concentration increased from 0.5 to $2.5 \%$ by weight in PVA films, the level of saturation also increases contributing to minimization of inter-particle distance between ZTA molecules. The chemical composition of the synthesized ZTA nanopowder was identified using EDX. The results are shown in Fig. 4. The elemental composition with respect to atomic $\%$ and weight $\%$ for ZTA nanopowder were also found using EDX. The weight $\%$ of oxygen, aluminium and zirconium in ZTA nanopowder were found to be 47.73 , 32.44 and 19.83 respectively. The atomic \% of oxygen, aluminium and zirconium in ZTA nanopowder were found to be $67.75,27.31$ and 4.94 respectively.

\subsection{DSC}

Polymer nanocomposite thin films were analysed for their thermal properties using DSC. Thermal data such as glass transition temperature $\left(T_{g}\right)$, melting temperature $\left(T_{m}\right)$ and heat of fusion $(\Delta \mathrm{H})$ were found out and the effect of nanofiller content on PVA thin films were analysed. DSC analysis was performed on a heat-cool-heat cycle. The heating curves and cooling curves are as shown in Fig. 5a and $b$ respectively. A detailed outline of the DSC data corresponding to these curves is as shown in Table 1. Once the sample was heated, different peaks were obtained due to the thermal decomposition of the sample.

The polymer nano composite films were analysed from 30 to $250^{\circ} \mathrm{C}$. Heating rate was carried out at $10^{\circ} \mathrm{C} / \mathrm{min}$. It is evident from Table 1. that, addition of ZTA into PVA matrix has led to a slight increase in $T_{g}$ and $T_{m}$ values. This phenomenon has probably occurred due to the strengthening of the polymer chains and enhanced intermolecular interaction between PVA and ZTA molecules. The increase in nanofiller content has led to slight decrease in the percentage crystallinity $\left(\% \mathrm{X}_{\mathrm{c}}\right)$ in the polymer nanocomposite films, which in turn has contributed to a slight increase in the crystallization temperature. Highest percentage crystallinity among the polymer nanocomposite films was observed for $1.5 \mathrm{wt} \%$ ZTA in PVA matrix with $29.55 \%$ crystallinity. Since PVA is a semi-crystalline polymer and ZTA is a crystalline ceramic, the polymer nanocomposite films tend to exhibit the properties of a crystalline polymer and hence affecting the crystallinity of the composite itself.

\subsection{AC conductivity}

Polymer nanocomposite films subjected to an applied electric field, which induces alternating conductivity within a sample due to the motion of charge carries. AC conductivity is measured using the Eq. (3) [21]:

$\sigma_{\mathrm{ac}}=(\mathrm{G} \cdot \mathrm{t}) / \mathrm{A}$

where $\mathrm{G}$ is the measured conductance $[\mathrm{S}]$, $\mathrm{t}$ is the film thickness $[\mathrm{m}]$, and $A$ is the effective cross-sectional area of the sample $\left[\mathrm{m}^{2}\right]$.

Figure 6 shows the dependence of AC conductivity on frequency. It is observed that at low frequencies up to $500 \mathrm{kHz}$, the conductivity of pure PVA films as well as PVA doped with ZTA nanocomposite films remained the same. The rise in conductivity at low frequencies was found to be negligible. Increase in frequency showed two interesting phenomenon occurring in the polymer nanocomposite films. Firstly, pure PVA film showed an exponential increase in $A C$ conductivity when the frequency increased beyond $0.5 \mathrm{MHz}$. Secondly, PVA doped with ZTA nanocomposite films showed negligible changes with increase in frequency. Even at frequencies as high as $5 \mathrm{GHz}$, PVA doped with ZTA nanocomposite films exhibited conductivity value as low as $7.53 \times 10^{-8} \mathrm{~S} / \mathrm{m}$. These results are true indication that PVA doped with ZTA nanocomposite films are best suited for electrical insulation applications. 


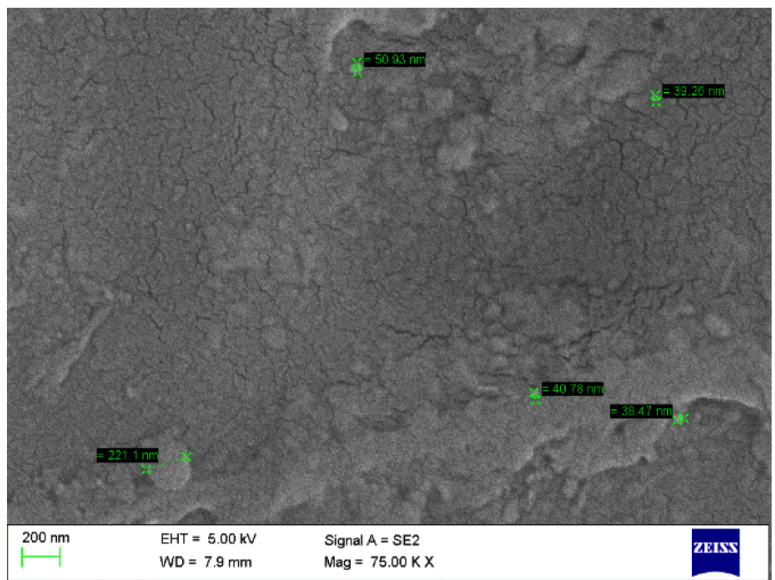

(a)

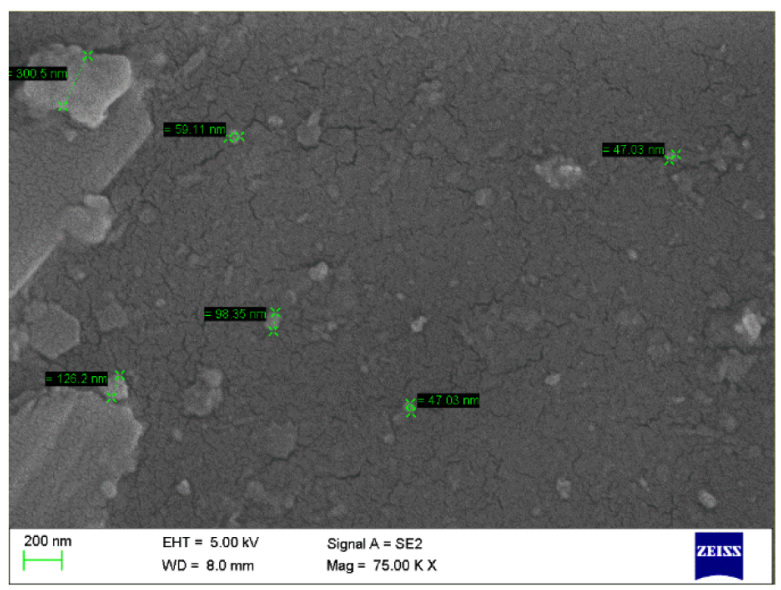

(c)

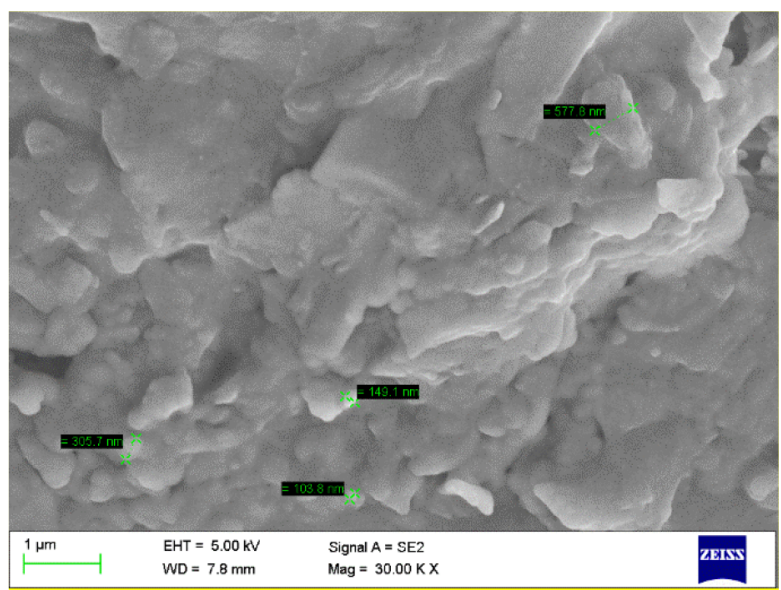

(e)

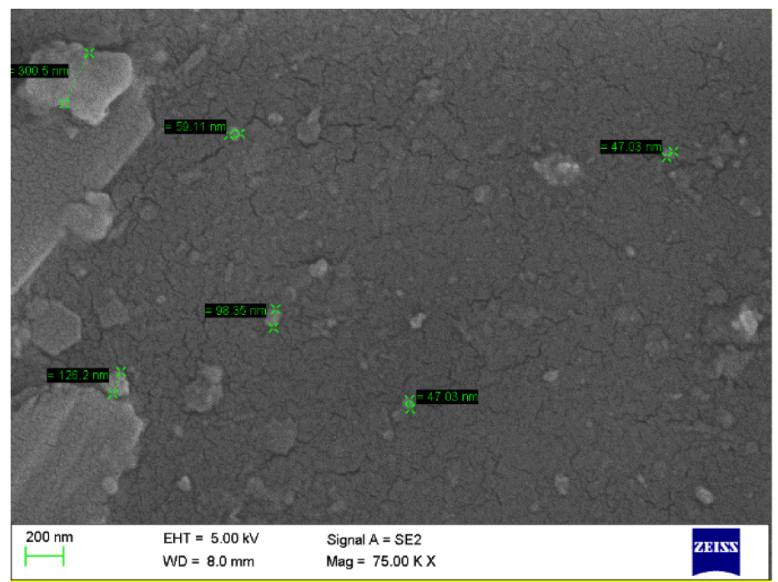

(b)

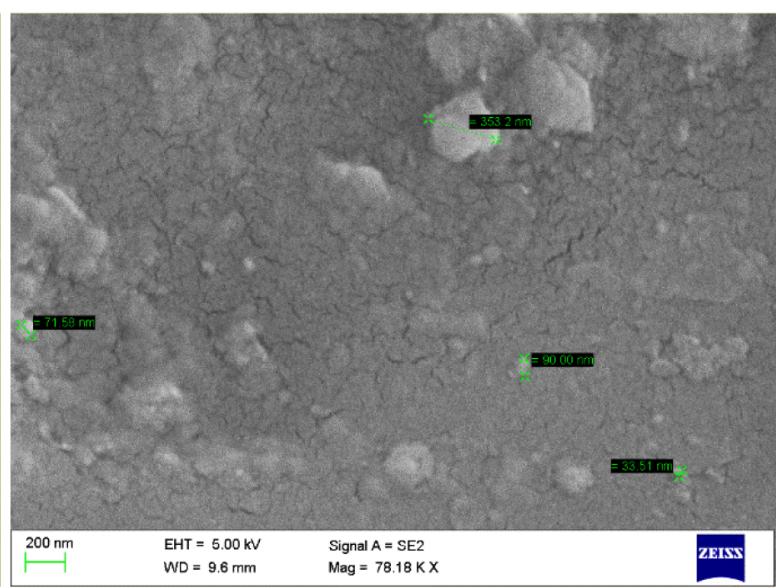

(d)

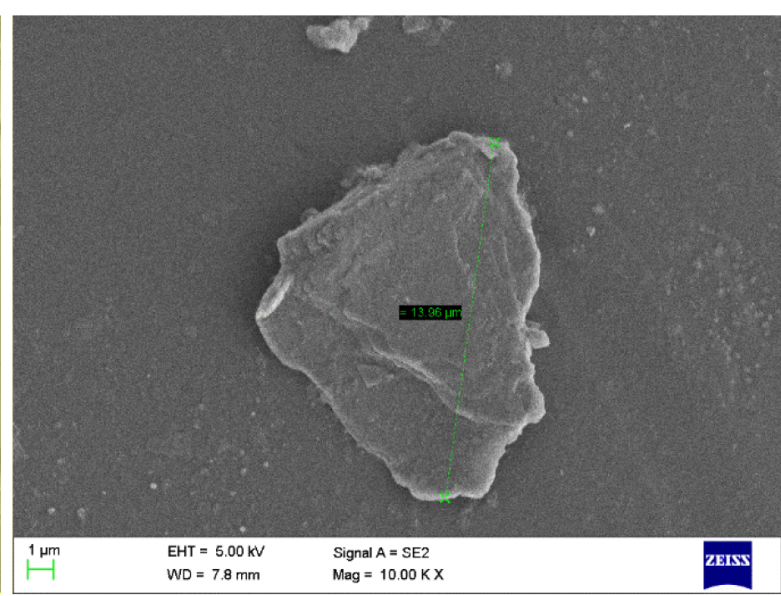

(f)

Fig. 3 SEM images of PVA loaded with ZTA nanoparticles at a 0 wt $\%, \mathbf{b} 0.5$ wt $\%$, c 1 wt $\%$, d 1.5 wt $\%$, e 2 wt $\%$ and f 2.5 wt $\%$ 


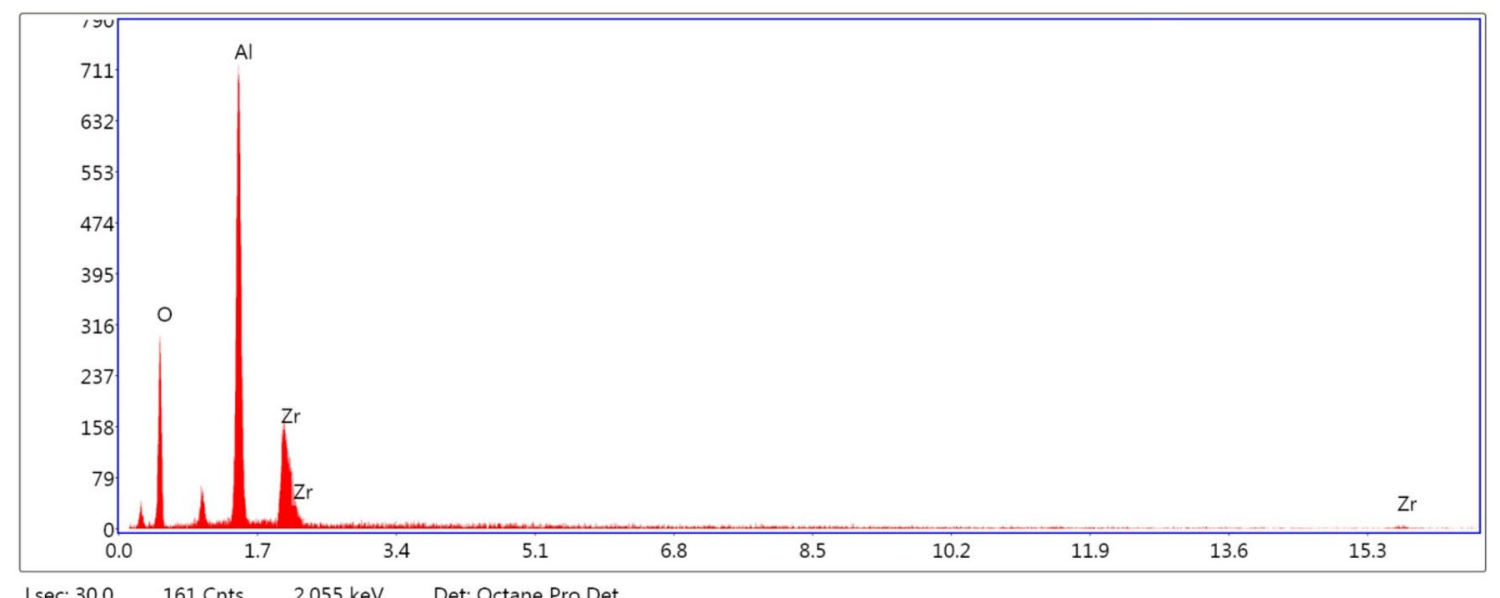

Fig. 4 Energy dispersive spectroscopy (EDS) of ZTA nanopowder

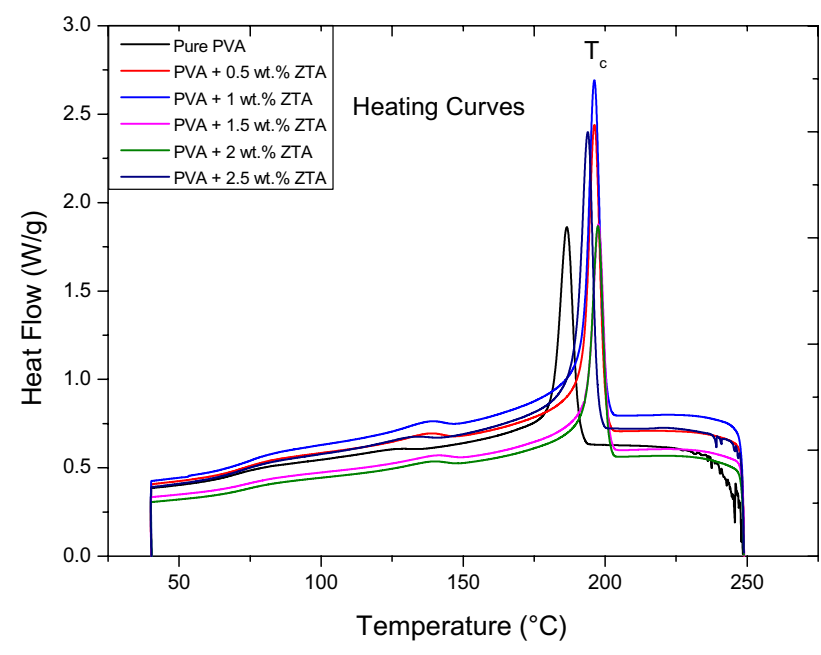

(a)

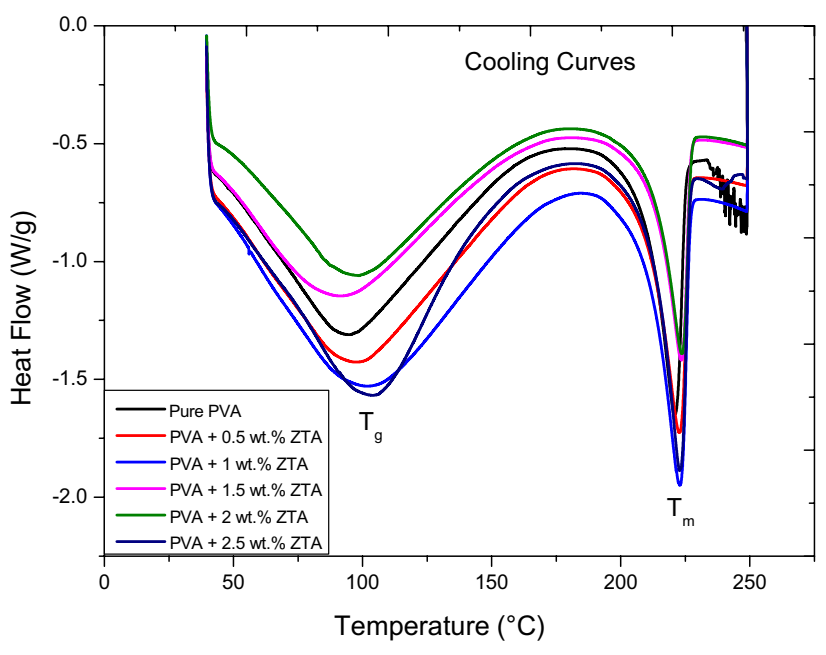

(b)

Fig. 5 a DSC heating curve and b DSC cooling curve for PVA-ZTA films

Table 1 Thermal data of PVAZTA nanocomposites

\begin{tabular}{lcccllll}
\hline \multicolumn{2}{l}{ Composition (wt\%) } & $\mathrm{T}_{\mathrm{g}}\left({ }^{\circ} \mathrm{C}\right)$ & $\mathrm{T}_{\mathrm{m}}\left({ }^{\circ} \mathrm{C}\right)$ & $\Delta \mathrm{H}_{\mathrm{m}}(\mathrm{J} / \mathrm{g})$ & $\Delta \mathrm{H}_{\mathrm{c}}(\mathrm{J} / \mathrm{g})$ & $\mathrm{T}_{\mathrm{c}}\left({ }^{\circ} \mathrm{C}\right)$ & $\% \mathrm{X}_{\mathrm{c}}$ \\
\cline { 1 - 5 } $\mathrm{PVA}$ & $\mathrm{ZTA}$ & & & & & & \\
\hline 100 & 0.0 & 95.35 & 220.68 & 76.7 & 48.77 & 186.5 & 36.41 \\
99.5 & 0.5 & 92 & 221.85 & 80.39 & 62.27 & 196.25 & 22.54 \\
99 & 1.0 & 103.2 & 222.37 & 90.26 & 65.22 & 196.22 & 27.74 \\
98.5 & 1.5 & 93.75 & 223.47 & 63.01 & 44.4 & 197.67 & 29.55 \\
98 & 2.0 & 98.6 & 223.56 & 63.16 & 49.7 & 197.51 & 21.31 \\
97.5 & 2.5 & 104.71 & 222.77 & 81.38 & 65.35 & 193.92 & 19.67 \\
\hline
\end{tabular}




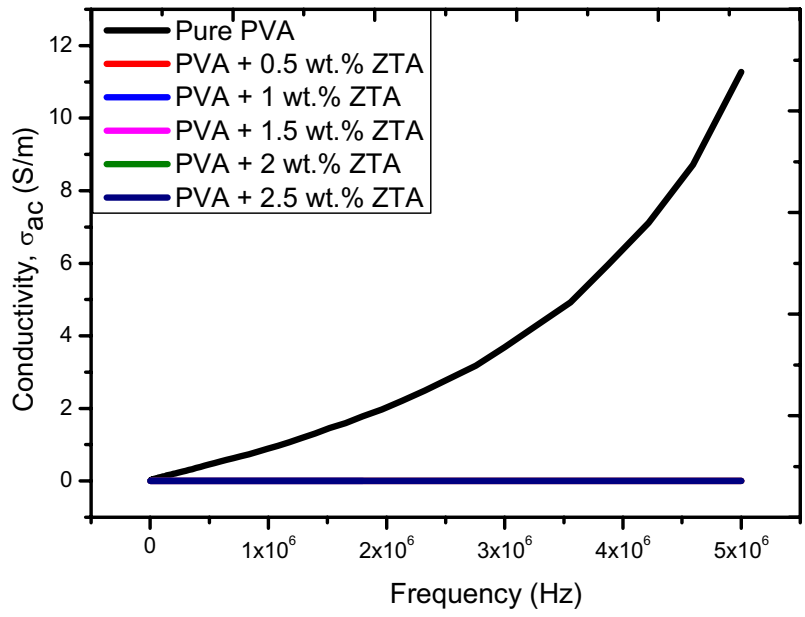

Fig. 6 AC conductivity of PVA-ZTA nanocomposites as a function of Frequency

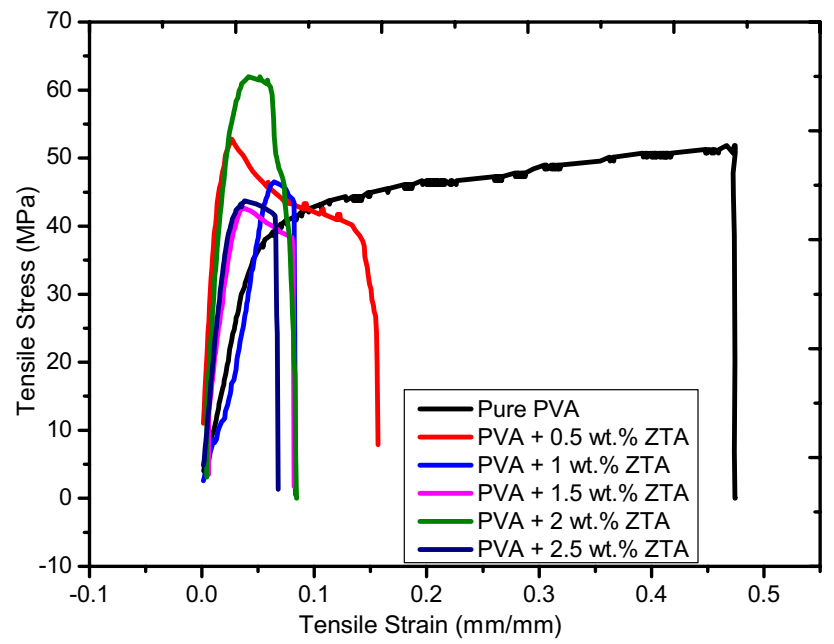

Fig. 7 Stress- Strain relationship of pure PVA film and for varying concentrations of ZTA with PVA

\subsection{Effect of ZTA nanoparticle concentration on mechanical properties of PVA films}

\subsubsection{Effect of filler loading on stress-strain relationship of PVA films}

Figure 7 depicts the relationship between stress and strain of pure PVA and PVA films doped with ZTA. It is clearly evident that, pure PVA film exhibits the highest elongation and highest energy at break-even point. Increase in ZTA content has gradually led to increase in tensile strength and the highest tensile strength is exhibited by PVA film doped with 2 wt $\%$ ZTA, further increase in ZTA concentration has further led to decrease in tensile strength and elongation at the break-even

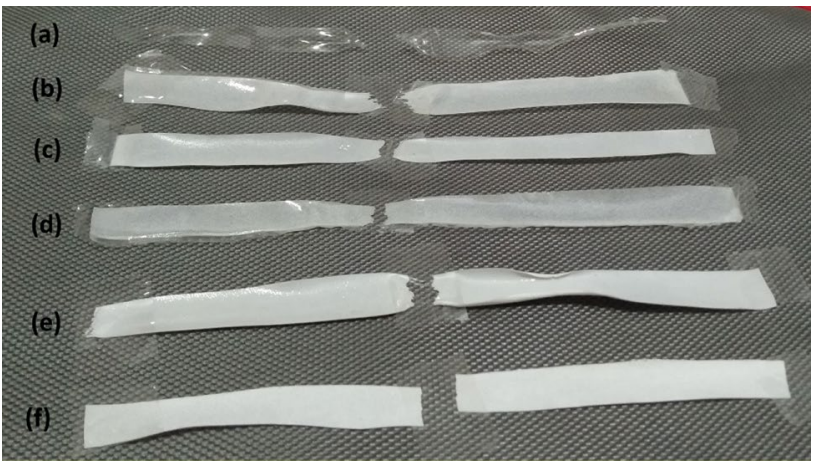

Fig. 8 Image of tested samples of PVA loaded with ZTA nanoparticles at a 0 wt $\%$, b 0.5 wt $\%$, c 1 wt $\%$, d 1.5 wt $\%$, e 2 wt $\%$ and f $2.5 \mathrm{wt} \%$

points. The enhanced mechanical properties may have been contributed due to very good interaction between PVA and ZTA molecules. The decrease in tensile strength value and elongation at break-even points may have probably occurred due to the saturation level of ZTA molecules in PVA matrix. Increase in ZTA loading in PVA has contributed to decrease in tensile strain. This might have occurred due to increase in the hardness in the polymer nanocomposite material. Chuankrerkkul et al. [27] have reported that, when ZTA nanopowder is reinforced into a polymer matrix, it enhances the hardness significantly in the parent material. In our case, the increase in hardness has further led to increase in the brittle nature and stiffness of the polymer nanocomposite. Figure 8 shows the image of the tested samples with varying concentrations of ZTA nanoparticles in PVA. The effect of ZTA content on various mechanical properties of films are summarized in Table 2.

\subsubsection{Effect of filler loading on Young's modulus}

Young's modulus of the all the polymer nanocomposite films are as shown in Fig. 9. Young's modulus was found to increase with increase in ZTA content in PVA matrix. Profound increase of $51.58 \%$ was observed just with $0.5 \mathrm{wt} \%$ addition of ZTA in the film, as the ZTA content kept on increasing, the value of Young's modulus also kept on increasing steeply. Highest increase of 304\% in Young's modulus was observed at 2 wt $\%$ addition of ZTA into PVA matrix. This exponential rise may have been contributed due to the intermolecular interaction between the ZTA molecules and PVA matrix. The strong adhesion between ZTA molecules and PVA may have resulted in the strong load bearing capacity of the films thereby increasing the Young's modulus. At $2.5 \mathrm{wt} \%$ loading of ZTA into PVA slight decrease in Young's modulus was observed, this may be due to the saturation level reached by the ZTA molecules 
Table 2 Effect of ZTA content on various mechanical properties of films

\begin{tabular}{lllllll}
\hline $\begin{array}{l}\text { ZTA content } \\
(\text { wt\%) }\end{array}$ & $\begin{array}{l}\text { Young's modu- } \\
\text { lus (MPa) }\end{array}$ & $\begin{array}{l}\text { Increase in Young's } \\
\text { modulus (\%) }\end{array}$ & Toughness (MPa) & $\begin{array}{l}\text { Decrease in } \\
\text { toughness (\%) }\end{array}$ & $\begin{array}{l}\text { Energy at break } \\
\text { even point (\%) }\end{array}$ & $\begin{array}{l}\text { Ultimate tensile } \\
\text { strength (MPa) }\end{array}$ \\
\hline $0.0^{\mathrm{a}}$ & 663.6 & - & 21.0 & - & 54.6 & 51 \\
0.5 & 1005.9 & 51.58 & 6.53 & 68.9 & 15.59 & 46 \\
1.0 & 1408.9 & 112.31 & 2.329 & 88.9 & 8.20 & 48 \\
1.5 & 1766 & 166.12 & 2.772 & 86.8 & 8.13 & 52.8 \\
2.0 & 2681 & 304 & 3.909 & 81.38 & 8.27 & 62.4 \\
2.5 & 2453 & 269.6 & 2.364 & 88.74 & 6.71 & 62 \\
\hline
\end{tabular}

${ }^{\mathrm{a}}$ Refers to pure PVA film

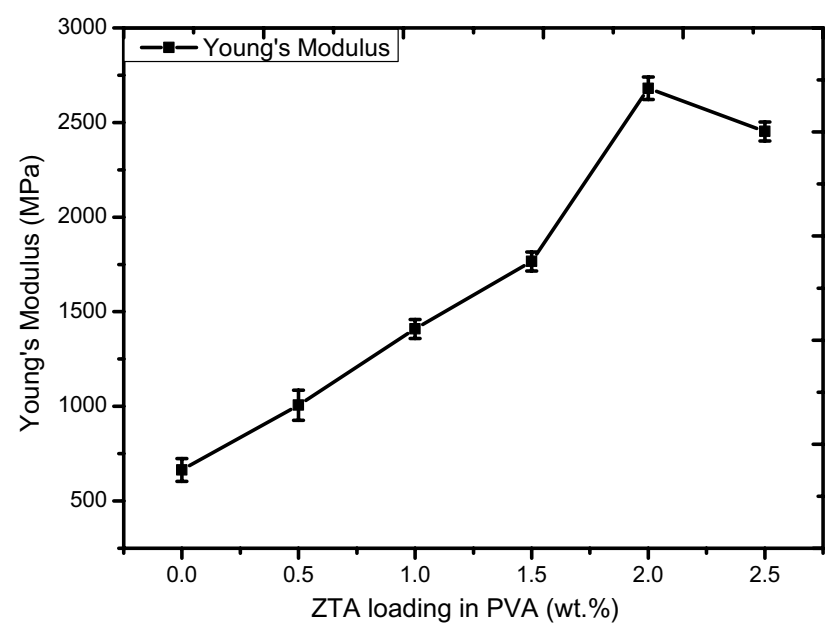

Fig. 9 Effect of ZTA content on Young's modulus of the film

in PVA. Juhasz et al. [28] studied the effect of filler content and particle size on the mechanical properties of the polymer composites. They found that, at low filler concentration in the composite, Young's modulus values also increased, as smaller particles provide greater surface area for interaction with the polymer resulting in an effective reinforcement, thereby enhancing the properties of the composite. Whereas, at higher filler loading, the particles tend to form agglomerates. These agglomerates tend to have a detrimental effect on the polymer composite since they act as failure initiation sites in the polymer composite resulting in crack propagation at low tensile strain [28]. The agglomerates also contribute to void formation in the polymer composite, they grow and deform the matrix, eventually leading to failure mechanisms at low tensile strain [28]. Suzuki et al. [23] also found that, increase in alumina content in PVA had a significant increase in Young's modulus values (184\% max.). The tensile strain also decreased with increase in alumina content in PVA. The percentage error as shown in Fig. 9 varied from 1 to $2 \%$.

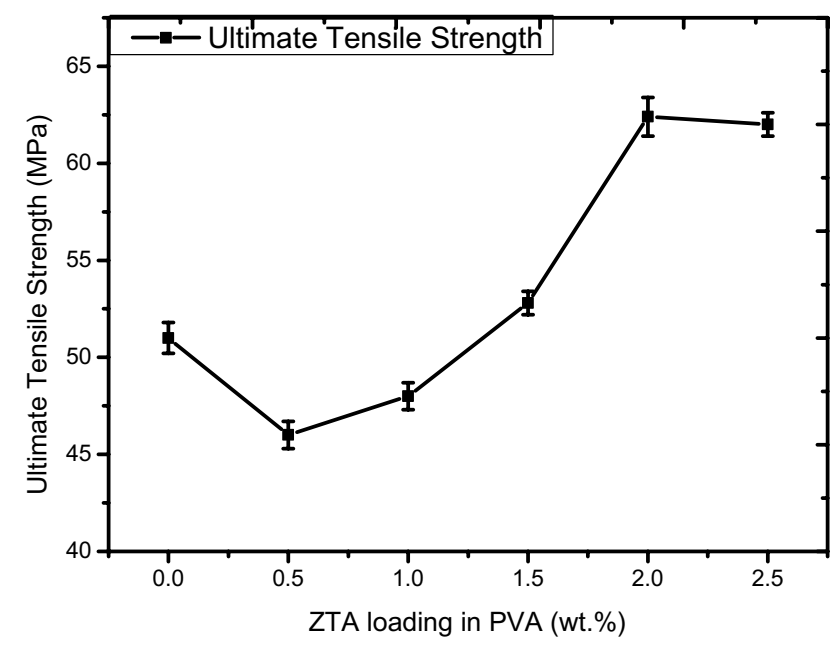

Fig. 10 Effect of ZTA content on ultimate tensile strength of the film

\subsubsection{Effect of filler loading on ultimate tensile strength of the film}

Figure 10 indicates the variation of ultimate tensile strength with ZTA loading in PVA films. Initial increase in ZTA loading in PVA films resulted in a slight increase in the ultimate tensile strength, as the concentration of ZTA increased from 1.5 to $2.5 \mathrm{wt} \%$ a profound increase in tensile strength values were observed. The highest tensile strength value of $62.4 \mathrm{MPa}$ was observed for $2 \mathrm{wt} \%$ ZTA loading in PVA films. Good interfacial tension between ZTA molecules and PVA matrix at $2 \mathrm{wt} \%$ may have probably attributed to increase in the tensile strength values. $A$ slight decrease in tensile strength was observed at $2.5 \mathrm{wt} \%$ ZTA loading in PVA films. Wang et al. [29] discussed the effect of particle size on mechanical properties of the composites. They found that, smaller the particle size, the polymer composites exhibit higher tensile strength. At filler concentration, especially for ceramic particles, there is a reduction in the tensile strength. This is due to the necking mechanism that occurs at higher filler concentrations, 


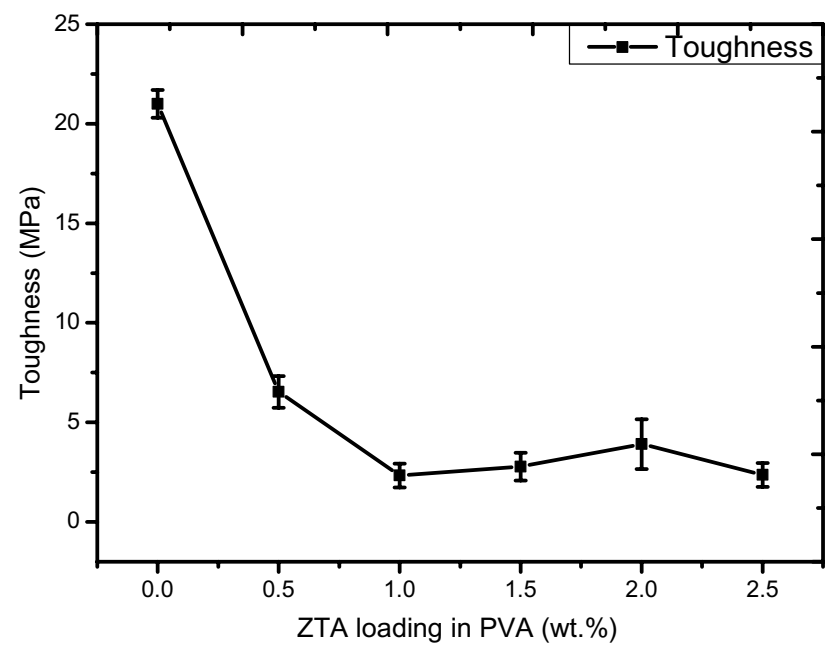

Fig. 11 Effect of ZTA loading on film toughness

subsequent fracture of polymer composites [29]. They [29] also observed that, with increase in filler loading the stress-strain curves became steeper and the polymer composite tends to lose its ductile properties and transforming it into a brittle material. Similar results were observed by Suzuki et al. [23]. They found a slight increase in tensile strength values with increase in alumina content in PVA. The percentage error as shown in Fig. 10 varied from 1 to $3.5 \%$.

\subsubsection{Effect of ZTA loading on film toughness}

Film toughness for ZTA in PVA matrix was calculated by the area under the curve of Tensile Stress v/s Tensile Strain graph $[17,18,20]$. Figure 11 shows the effect of ZTA loading on film toughness. Film toughness was found to decrease very steeply with increase in ZTA loading. Increase in ZTA concentration did not contribute to further enhancement in the film toughness. This may be probably due to the high stability of the tetragonal phase of zirconia molecules which is achieved by doping ZTA into PVA. Since the amount of stability in tetragonal phase has inverse relationship with film toughness [30], the increase in ZTA content in the polymer nanocomposite films has led to decrease in the film toughness. Another reason for decrease in film toughness may be due to ceramic property of the reinforcing material [23]. Similar decrease in tensile strain values and reduction in film toughness was observed when alumina was reinforced into PVA films [23]. The percentage error as shown in Fig. 11 varied from 1 to $3 \%$.

\section{Conclusion}

ZTA nano-powders were synthesized by economically viable solution combustion route. The synthesized nanopowders were doped into PVA and the effect of doping concentration on PVA were studied. The nanocomposite films were examined for SEM, EDX, XRD and DSC before investigating the electrical properties. $X R D$ results revealed that zirconia existed in tetragonal phase and alumina existed hexagonal phase. Crystal size was analysed using Debye-Scherrer formula and average particle size of ZTA nanopowder was found to be around $30-50 \mathrm{~nm}$. Doping ZTA into PVA matrix showed an interesting transformation of the polymer from semi-crystalline to crystalline nature. SEM results showed that, ZTA nanoparticles were distributed homogenously and the level of agglomeration in films was directly dependent on the increase in doping concentration of ZTA nanopowder. EDS results revealed the chemical composition of ZTA nanopowder. Thermal analysis using DSC showed that the crystallization temperature of the nanocomposite films kept on increasing with increase in ZTA concentration in the films. This is probably due to the crystalline nature of ZTA. AC conductivity measurements were carried out using impedance analyser. The results revealed that conductivity decreased with increase in frequency and doping concentration. Pure PVA exhibited semiconducting behaviour. Increase in ZTA concentration in PVA transformed the semiconducting nature of PVA to insulating material. At high frequencies specially in the range of $1.25 \mathrm{GHz}$ to $5 \mathrm{GHz}$, all the nano polymer composites exhibited excellent dielectric properties indicating that ZTA doped polymer nanocomposites are suitable for electrical insulation applications. Enhanced mechanical properties such as Young's modulus and ultimate tensile strength were observed with ZTA loading in PVA matrix. Best results for Young's modulus and ultimate tensile strength were observed for $2 \mathrm{wt} \%$ loading of ZTA in PVA matrix, the values were found to be $2681 \mathrm{MPa}$ and 62.4 MPa respectively. Film toughness was found to decrease with increase in the nano filler loading due to the stabilized tetragonal phase of zirconia in the ZTA nanopowder. These results suggest that ZTA is a promising nanomaterial for future electrical insulation and mechanical applications.

Acknowledgements The authors are very grateful to Department of Science and Technology, for providing financial assistance under Women Scientist A-Scheme to carry out the entire project. Project Sanction No. SR/WOS-A/ET-16/2017. They are also thankful to Department of Chemical Engineering, MSRIT, Bangalore, for the technical support to carry out the research work. Electrical testing was performed at CeNSE lab, IISC, Bangalore. Mechanical properties testing was carried out TERI, Bangalore. 


\section{Compliance with ethical standards}

Conflict of interest The authors declare that they have no conflict of interest.

\section{References}

1. Yildiz Ö, Yilmaz M (2017) Synthesis and characterisation of nano powders for production of zirconia toughened alumina bioceramic implant materials. Int J Biomed Nanosci Nanotechnol 3(4):320-334. https://doi.org/10.1504/IJBNN.2017.085446

2. Sarkar D, Mohapatra D, Ray S, Bhattacharyya S, Adak S, Mitra N (2007) Synthesis and characterization of sol-gel derived $\mathrm{ZrO}_{2}$ doped $\mathrm{Al}_{2} \mathrm{O}_{3}$ nanopowder. Ceram Int 33(7):1275-1282. https:// doi.org/10.1016/j.ceramint.2006.05.002

3. Kyomoto M, Shoyama Y, Saiga K, Moro T, Ishihara K (2018) Reducing fretting-initiated crevice corrosion in hip simulator tests using a zirconia-toughened alumina femoral head. J Biomed Mater Res Part B Appl Biomater 106(8):2815-2826. https://doi. org/10.1002/jbm.b.34062

4. Shishkovsky I, Yadroitsev I, Bertrand P, Smurov I (2007) Aluminazirconium ceramics synthesis by selective laser sintering/melting. Appl Surf Sci 254(4):966-970. https://doi.org/10.1016/j. apsusc.2007.09.001

5. Perrichon A, Reynard B, Gremillard L, Chevalier J, Farizon F, Geringer J (2017) A testing protocol combining shocks, hydrothermal ageing and friction, applied to Zirconia Toughened Alumina (ZTA) hip implants. J Mech Behav Biomed Mater 65:600-608. https://doi.org/10.1016/j.jmbbm.2016.09.019

6. Reyes-Rojas A, Dominguez-Rios C, Garcia-Reyes A, AguilarElguezabal A, Bocanegra-Bernal MH (2018) Sintering of carbon nanotube-reinforced zirconia-toughened alumina composites prepared by uniaxial pressing and cold isostatic pressing. Mater Res Express 5(10):105602. https://doi.org/10.1088/2053-1591/ aada35

7. Cassir M, Goubin F, Bernay C, Vernoux P, Lincot D (2002) Synthesis of $\mathrm{ZrO}_{2}$ thin films by atomic layer deposition: growth kinetics, structural and electrical properties. Appl Surf Sci 193(1-4):120128. https://doi.org/10.1016/S0169-4332(02)00247-7

8. Bastianini A, Battiston GA, Gerbasi R, Porchia M, Daolio S (1995) Chemical vapor deposition of $\mathrm{ZrO}_{2}$ thin films using $\mathrm{Zr}$ (NEt2) 4 as precursor. J Phys IV 5:C5-525. https://doi.org/10.1051/jphys col:1995561

9. Sequeira S, Fernandes MH, Neves N, Almeida MM (2017) Development and characterization of zirconia-alumina composites for orthopedic implants. Ceram Int 43(1):693-703. https://doi. org/10.1016/j.ceramint.2016.09.216

10. Fan J, Lin T, Fangxuan H, Yi Yu, Ibrahim M, Zheng R, Huang S, Ma $J(2017)$ Effect of sintering temperature on microstructure and mechanical properties of zirconia-toughened alumina machinable dental ceramics. Ceram Int 43(4):3647-3653. https://doi. org/10.1016/j.ceramint.2016.11.204

11. Ikeda J, Takayuki Murakami T, Sasaki T, Shimozono Y Shouyama, Iwamoto M (2017) Wear and corrosion resistance of low temperature degradation free ZTA for artificial joint. Key Eng Mater 720:296-300. https://doi.org/10.4028/www.scientific.net/ KEM.720.296

12. Aruna ST, Rajam KS (2004) Mixture of fuels approach for the solution combustion synthesis of $\mathrm{Al}_{2} \mathrm{O}_{3}-\mathrm{ZrO}_{2}$ nanocomposite. Mater Res Bull 39(2):157-167. https://doi.org/10.1016/j.mater resbull.2003.10.005
13. Tulliani J-M, Lombardi $M$, Palmero $P$, Fornabaio $M$, Gibson LJ (2013) Development and mechanical characterization of novel ceramic foams fabricated by gel-casting. J Eur Ceram Soc 33(9):1567-1576. https://doi.org/10.1016/j.jeurcerams oc.2013.01.038

14. Nagashima M, Maki K, Hayakawa M (2001) Fabrication of Al2O3/ ZrO2 micro/nano-composite prepared by high energy ball milling. Mater Trans 42(6):1119-1123. https://doi.org/10.2320/ matertrans.42.1119

15. Wang X, Tian J, Xuegang Yu, Shan Y, Liu Z, Yin Y (2008) Effect of microstructure on the fracture behavior of micro-nano ZTA composite. Mater Chem Phys 112(1):213-217. https://doi. org/10.1016/j.matchemphys.2008.05.041

16. Sarkar D, Adak S, Mitra NK (2007) Preparation and characterization of an $\mathrm{Al}_{2} \mathrm{O}_{3}-\mathrm{ZrO}_{2}$ nanocomposite, part l: powder synthesis and transformation behavior during fracture. Compos Part $A$ Appl Sci Manuf 38(1):124-131. https://doi.org/10.1016/j.compo sitesa.2006.01.005

17. Srikanth C, Madhu GM, Bhamidipati H, Srinivas S (2019) The effect of CdO-ZnO nanoparticles addition on structural, electrical and mechanical properties of PVA films. AIMS Mater Sci 6(6):1107-1123. https://doi.org/10.3934/matersci.2019.6.1107

18. Rao JK, Raizada A, Ganguly D, Mankad MM, Satyanarayana SV, Madhu GM (2015) Enhanced mechanical properties of polyvinyl alcohol composite films containing copper oxide nanoparticles as filler. Polym Bull 72(8):2033-2047. https://doi.org/10.1007/ s00289-015-1386-4

19. Rashmi SH, Raizada A, Madhu GM, Kittur AA, Suresh R, Sudhina HK (2015) Influence of zinc oxide nanoparticles on structural and electrical properties of polyvinyl alcohol films. Plast, Rubber Compos 44(1):33-39. https://doi.org/10.1179/1743289814 Y.0000000115

20. Rashmi SH, Soumyashree A, Shrusti S, Shivani S, Aamir D, Kittur AA, Sudhina HK, Koteswararao J, Madhu GM (2018) Structural mechanical and electrical property evaluation of nano cadmium oxide polyvinyl alcohol composites. Int J Plast Technol 22(1):4155. https://doi.org/10.1007/s12588-018-9201-321

21. Koteswararao J, Abhishek R, Satyanarayana SV, Madhu GM, Venkatesham V (2016) Influence of cadmium sulfide nanoparticles on structural and electrical properties of polyvinyl alcohol films. Express Polym Lett 10(11):883-894. https://doi.org/10.3144/ expresspolymlett.2016.83

22. Rao JK, Raizada A, Ganguly D, Mankad MM, Satayanarayana SV, Madhu GM (2015) Investigation of structural and electrical properties of novel CuO-PVA nanocomposite films. J Mater Sci 50(21):7064-7074. https://doi.org/10.1007/s10853-015-9261-0

23. Suzuki F, Onozato K, Kurokawa Y (1990) A formation of compatible poly (vinyl alcohol)/alumina gel composite and its properties. J Appl Polym Sci 39(2):371-381. https://doi.org/10.1002/ app.1990.070390214

24. Panda PK, Ramakrishna S (2007) Electrospinning of alumina nanofibers using different precursors. J Mater Sci 42(6):21892193. https://doi.org/10.1007/s10853-007-1581-2

25. Bhaduri S, Bhaduri SB, Zhou E (1998) Auto ignition synthesis and consolidation of $\mathrm{Al}_{2} \mathrm{O}_{3}-\mathrm{ZrO}_{2}$ nano/nano composite powders. J Mater Res 13(1):156-165. https://doi.org/10.1557/ JMR.1998.002115

26. Vasylkiv O, Sakka Y, Skorokhod VV (2003) Low-temperature processing and mechanical properties of zirconia and zirconia-alumina nanoceramics. J Am Ceram Soc 86(2):299-304. https://doi. org/10.1111/j.1151-2916.2003.tb00015

27. Chuankrerkkul N, Somton K, Wonglom T, Dateraksa K, Laoratanakul P (2016) Physical and mechanical properties of zirconia toughened alumina (ZTA) composites fabricated by powder injection moulding. Chiang Mai J Sci 43:375-380 
28. Juhasz JA, Best SM, Brooks R, Kawashita M, Miyata N, Kokubo T, Nakamura T, Bonfield W (2004) Mechanical properties of glass-ceramic A-W-polyethylene composites: effect of filler content and particle size. Biomaterials 25(6):949-955. https:// doi.org/10.1016/j.biomaterials.2003.07.005

29. Wang M, Joseph R, Bonfield W (1998) Hydroxyapatite-polyethylene composites for bone substitution: effects of ceramic particle size and morphology. Biomaterials 19(24):2357-2366. https:// doi.org/10.1016/S0142-9612(98)00154-9
30. Magnani G, Brillante A (2005) Effect of the composition and sintering process on mechanical properties and residual stresses in zirconia-alumina composites. J Eur Ceram Soc 25(15):33833392. https://doi.org/10.1016/j.jeurceramsoc.2004.09.025

Publisher's Note Springer Nature remains neutral with regard to jurisdictional claims in published maps and institutional affiliations. 